\title{
miR-506 Inhibits Epithelial-to-Mesenchymal Transition and Angiogenesis in Gastric Cancer
}

Zhen $\mathrm{Li}_{1}{ }^{* \dagger}$ Zhimin Liu, ${ }^{\dagger}$ Suwei Dong, ${ }^{* \dagger}$ Jianhua Zhang, ${ }^{\dagger}$ Jing Tan, ${ }^{\dagger}$ Ying Wang, ${ }^{\dagger}$ Chunlei Ge, ${ }^{\dagger}$ Ruilei Li, ${ }^{\dagger}$ Yuanbo Xue, ${ }^{\dagger}$ Mei Li, ${ }^{\S}$ Weiwei Wang, ${ }^{\top}$ Xudong Xiang, ${ }^{\top}$ Jinyan Yang, ${ }^{\dagger}$ Haiyan Ding, ${ }^{\dagger}$ Tao Geng, ${ }^{\dagger}$ Kaitai Yao, ${ }^{*}$ and Xin Song ${ }^{* \dagger}$

From the Cancer Research Institute of Southern Medical University, * Guangzhou; and the Cancer Biotherapy Center ${ }^{\dagger}$ and the Departments of General Surgery, ${ }^{\ddagger}$ Pathology, ${ }^{\S}$ and Thoracic Surgery, ${ }^{\top}$ The Third Affiliated Hospital of Kunming Medical University (Tumor Hospital of Yunnan Province), Kunming, People's Republic of China

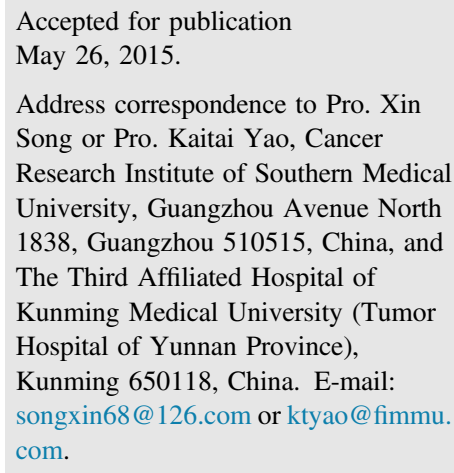

Gastric cancer is one of the most common malignancies in developing countries. We examined the possible role of miR-506 in gastric cancer, investigated its associations with the clinical outcomes of gastric cancer patients, and explored its potential role in angiogenesis and the metastasis of gastric cancer cells. We found that miR-506 expression was a useful marker for stratifying patients from early to advanced clinical stages and for overall survival prediction. miR-506 overexpression inhibited the epithelial-to-mesenchymal transition of gastric cancer cells; however, depletion of miR-506 promoted it. In addition, miR-506 suppressed gastric cancer angiogenesis and was associated with decreased matrix metalloproteinase- 9 expression. We also found that ETS1 was a miR-506 target, and it was expressed in $71.10 \%$ of gastric cancer tissue samples. Moreover, ETS1 expression was associated with matrix metalloproteinase- 9 expression $(P<0.001)$. In conclusion, miR-506 was identified as an ETS1 targeting suppressor of metastatic invasion and angiogenesis in gastric cancer. (Am J Pathol 2015, 185: 2412-2420; http:// dx.doi.org/10.1016/j.ajpath.2015.05.017)
Although the incidence of gastric cancer has declined by $>80 \%$ in developed countries during the past 50 years, it remains one of the most significant health problems in the developing countries. ${ }^{1-3}$ In China, gastric cancer is the third leading cause of cancer-related death. ${ }^{4}$ Gastric cancer patients in China and most other developing countries are usually diagnosed during the advanced stages of the disease. Because of the high likelihood of metastasis in advanced gastric cancer, the characteristically late diagnosis contributes to the high rate of gastric cancer mortality. ${ }^{5}$ A better understanding of the molecular mechanisms underlying gastric cancer development and progression could help the development of novel approaches for the early detection and treatment of this type of cancer.

In recent years, post-transcriptional regulation has been intensively studied as a phenotype-level control mechanism of gene expression regulation. ${ }^{6}$ Among the posttranscriptional regulators with metastatic activity, one of the most studied classes is small noncoding RNAs. ${ }^{7-9}$ miRNAs are a class of small noncoding RNAs that regulate gene expression by binding to the $3^{\prime}$-untranslated region ( $3^{\prime}$-UTR) of target genes. miRNA regulation results in mRNA degradation and the inhibition of protein translation. ${ }^{10}$ Although an increasing number of studies examining different miRNAs in various pathophysiological settings have been reported, ${ }^{11,12}$ only a few of these interactions have been validated experimentally.

In this study, we demonstrate that miR-506 is deregulated in metastatic gastric cancer cell lines and that miR-506 deficiency is associated with poor overall gastric cancer patient survival. In addition, we report that miR-506

\footnotetext{
Supported, in part, by National Natural Science Foundation of China grants $30671904,81360322,81060185,81260307$, and 81470005, Scientific Research Project of the Department of Education of Yunnan Province grant 2011J043, Leading Talent of Health Systems of Yunnan Province grant L-201213, and National Clinical Key Specialty Construction Projects of Oncology of the National Health and Family Planning Commission of China (awarded to the Tumor Hospital of Yunnan Province: 2013-2014).

Z. Li, Z. Liu, and S.D. contributed equally to this work.

Disclosures: None declared.
} 
overexpression in gastric cancer inhibits endothelial cell angiogenesis and metastatic invasion by targeting the protooncogene ETS1, which plays several important roles in the angiopoietic and developmental processes of cancer cells. ${ }^{13,14}$ Therefore, we hypothesized that miR-506 targeting of ETS1 influences the angiogenic activity of endothelial cells and gastric cancer metastasis.

\section{Materials and Methods}

\section{Patients and Tissue Specimens}

For miR-506 detection, gastric cancer specimens were obtained from 109 patients who underwent surgery without any radiotherapy, chemotherapy, or biotherapy from The Department of Pathology, The Third Affiliated Hospital, Kunming Medical University (Kunming, China), from January 2008 through November 2013. After collection, samples were immediately frozen and kept in liquid nitrogen until further use. Follow-up data were collected from 84 of the 109 patients. For immunohistochemistry, specimens from 173 gastric cancer patients were collected from The Department of Pathology, The Third Affiliated Hospital, Kunming Medical University, from January 2003 through November 2008. For all patients, detailed clinicopathological data were collected, including sex, age, TNM staging, and clinical staging, as defined according to the criteria of the American Joint Commission on Cancer (seventh edition). ${ }^{15}$ Eight normal gastric tissue samples were obtained from macroscopically uninvolved areas 2 to $3 \mathrm{~cm}$ away from the benign nodules of patients with stomach leiomyoma $(n=5)$ or adenomyoepithelioma ( $n=3)$ who underwent surgical resection. All normal gastric tissues were histopathologically assessed and were morphologically normal. This study was approved by the Ethics Committee of The Third Affiliated Hospital, Kunming Medical University, and all patients provided written informed consent.

\section{Real-Time PCR}

For miRNA expression analysis, frozen tissue specimens were ground into powder in liquid nitrogen. Total RNA was extracted from 50 to $100 \mathrm{mg}$ of the powdered tissue using TRIzol reagent (Invitrogen, Carlsbad, CA) and reverse transcribed into cDNA using Superscript II reverse transcriptase (Invitrogen), according to the manufacturer's protocols. Real-time PCR for miR-506 was performed in a reaction mixture containing SYBR Premix Ex Taq (Takara Bio Inc., Shiga, Japan). Quantitation of miRNAs was performed using an ABI 7500 system (Life Technologies, Grand Island, NY), according to the manufacturer's protocol. All PCRs were performed in triplicate, and a relative quantitative method was applied using the averaged $\Delta \mathrm{C}_{\mathrm{T}}$ from the normal tissues or untreated cells. The endogenous control was U6.

\section{Cell Lines and Culture}

Primary cultures of normal gastric epithelial cells were established from fresh specimens of the adjacent noncancerous gastric tissue taken from an area over $10 \mathrm{~cm}$ from the cancerous tissue. The human gastric cancer cell lines AGS, BGC-823, HGC-27, Kato-III, SGC-7901, MKN45, and MGC-803 were supplied and authenticated by the Cell Bank of Shanghai Institute of Cell Biology and the Chinese Academy of Sciences (Shanghai, China). Cell lines were grown in RPMI 1640 medium (Invitrogen) supplemented with $10 \%$ fetal bovine serum (Invitrogen), $100 \mathrm{U} / \mathrm{mL}$ penicillin, and $100 \mu \mathrm{g} / \mathrm{mL}$ streptomycin (Sigma, St. Louis, $\mathrm{MO})$ in a humidified $5 \% \mathrm{CO}_{2}$ atmosphere at $37^{\circ} \mathrm{C}$.

\section{Construction and Transfection of Expression Vectors Containing miR-506 Inhibitors and Mimics}

The miR-506 mimics, inhibitors, and negative control were purchased from RiboBio Co Ltd (Guangzhou, China). For gene transfection, gastric cancer cells were grown overnight in the logarithmic growth phase and transiently transfected with miR-506 mimic, inhibitor, or negative control vectors using Lipofectamine 2000 (Invitrogen) for 48 hours, according to the manufacturer's protocol, before being subjected to analyses.

\section{Protein Extraction and Western Blot Analysis}

Total cellular protein was extracted from gastric cancer cells with or without miRNA transfection using a lysis buffer containing protease and phosphatase inhibitors. After protein quantification using a BCA Protein Assay Kit (Pierce 23,227; Thermo Fisher Scientific Inc., Rockford, IL), equal amounts of protein samples $(50 \mathrm{~g})$ were separated using SDS-PAGE and then transferred onto a $0.22-\mu \mathrm{m}$ polyvinylidene difluoride membrane (ISEQ00010; Millipore, Billerica, MA). For Western blot analysis, membranes were first incubated with $8 \%$ skimmed milk/Tris-buffered saline/Tween 20 at room temperature for 4 hours and then with a primary antibody overnight at $4^{\circ} \mathrm{C}$. The following primary antibodies were used: mouse monoclonal anti-E-cadherin (sc-59778; Santa Cruz Biotechnology Inc., Dallas, TX), mouse monoclonal anti-vimentin (sc-6260; Santa Cruz Biotechnology Inc.), and rabbit polyclonal anti- $\beta$-actin (ab8227; Abcam, Cambridge, UK). The following day, membranes were washed three times with Tris-buffered saline/Tween 20 and incubated for 1 hour with a secondary antibody (1:10,000 dilution for anti-mouse horseradish peroxidase-linked antibody $7074 ; 1: 20,000$ dilution for anti-rabbit horseradish peroxidase-linked antibody 7074; Cell Signaling Technology, Danvers, MA) and then detected using an ECL kit (RPN2235; Amersham ECL Select; GE Healthcare Life Sciences, Buckinghamshire, UK). Data were quantified using ImageJ software version $1.48 \mathrm{u}$ (NIH, Bethesda, MD; http://imagej.nih.gov/ij) and $\beta$-actin or glyceraldehyde-3-phosphate dehydrogenase levels. 


\section{Wound-Healing Assay}

SGC-7901 or BGC-823 cells were seeded at $2 \times 10^{6}$ cells per well in 6-well plates, grown to $100 \%$ confluency, and then kept in serum-free RPMI 1640 medium for 24 hours. Next, a wound was generated across the cell monolayer using a $100-\mu \mathrm{L}$ plastic pipette tip. Cell migration into the wound area was inspected under an inverted microscope for up to 24 hours after scarification. Quantitative analysis of the wound closure was calculated by measuring the initial width of the wound and the final width of the wound and calculating the distance of wound closure.

\section{Matrigel Invasion Assay}

SGC-7901 or BGC-823 cells were seeded at $5 \times 10^{4}$ cells per well with serum-free medium into the upper Matrigel invasion chamber (Corning Inc., Corning, NY), the filter of which was precoated with Matrigel (Sigma-Aldrich Shanghai Trading Co. Ltd., Shanghai, China) and the lower chamber of which was filled with a medium containing $10 \%$ fetal bovine serum as a chemoattractant. The cells were cultured for 20 hours and, at the end of the experiment, the cells remaining in the upper chamber were carefully removed and the cells that had invaded the bottom of the membrane were fixed and stained with hematoxylin. Quantification was performed by counting the stained cells.

\section{Three-Dimensional Cell Culture}

Matrigel was dissolved at $4{ }^{\circ} \mathrm{C}$ overnight and then used to coat 24 -well plates $(100 \mu \mathrm{L}$ per well). After a 30 -minute incubation at $37^{\circ} \mathrm{C}, \mathrm{BGC}-823$ or SGC-7901 cells $\left(2 \times 10^{4}\right)$ suspended in $2 \%$ liquid Matrigel were inoculated into the Matrigel-coated wells. Cells were then grown in a cell culture box for 10 to 14 days, and fresh culture medium was added every 3 to 4 days.

\section{Tube Formation Assay}

Cells $\left(5 \times 10^{4}\right.$ viable cells per well $)$ were seeded onto a 48 -well polystyrene plate coated with Matrigel $(120 \mu \mathrm{L}$ per well), which had been incubated at $37^{\circ} \mathrm{C}$ and $5 \% \mathrm{CO}_{2}$ for 30 minutes. Six hours after seeding, representative phasecontrast images were obtained at $\times 4$ magnification.

\section{CAM Assay}

Fertilized white leghorn chicken eggs were incubated in an incubator at $37^{\circ} \mathrm{C}$ with $60 \%$ humidity. A small window was made in the shell on day 7 of chick embryo development under aseptic conditions. The window was resealed with sterile adhesive tape, and the eggs were returned to the incubator until day 11 of chick embryo development. On day $11,10-\mu \mathrm{L}$ cell suspensions of BGC-823 or SGC-7901 cells were placed on top of the chick chorioallantoic membrane (CAM), and the eggs were resealed and returned to the incubator for 72 hours until day 14 ( $n=6$ chicken embryos per cell line). Fixation was
A
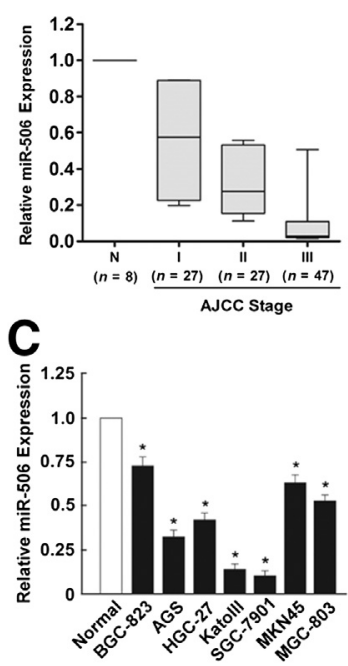

Figure 1 Correlation between miR-506 expression, the prognosis of gastric cancer patients, and the motility of gastric cancer cells. A: The clinical significance of miR-506 expression in patients with gastric cancer. miR-506 expression in normal gastric tissue was used as a control. B: KaplanMeier overall survival curves for gastric cancer patients with low and high miR-506 expression. Patients were divided into two sets on the basis of miR506 expression (greater or less than the mean, $P<0.05$ ). C: miR-506 expression in gastric cancer cell lines. miR-506 expression in normal gastric epithelial cells was used as a control. D: Invasion assay in gastric cancer cell lines in vitro. Cells were allowed to invade through Matrigel toward serum for 24 hours. The cells that invaded each filter were counted, and the number was plotted for each cell line. Error bars represent means \pm SD. ${ }^{*} P<0.05$. AJCC, American Joint Commission on Cancer (seventh edition).

performed using formaldehyde and acetone (1:1), and prefixation was performed for 15 minutes through the window. The transplanted tumors and surrounding chorioallantoic membrane were integrally unloaded for observation and imaging.

\section{Gelatin Zymography Assay}

For the gelatin zymography assay, proteins were extracted from the cultured supernatant of the BGC-823 or SGC-7901 gastric cancer cells, with or without miRNA transfection. Equal amounts of protein samples $(30 \mu \mathrm{g})$ were separated by electrophoresis under denaturing conditions (SDS-PAGE). After electrophoresis, the gel was washed four times in elutriant. After shock rinsing twice, the gel was incubated for 48 hours at $37^{\circ} \mathrm{C}$. Next, after the gel was rinsed three times, it was stained using SimplyBlue Safestain (Invitrogen) and incubated for 3 hours at room temperature under gentle agitation. Next, SimplyBlue SafeStain was removed, and the gel was destained according to the manufacturer's instructions. The gel was scanned with an image analysis system (Quantity One; Bio-Rad, Hercules, CA).

\section{Luciferase Assay}

TargetScan (http://www.targetscan.org, last accessed May 20,2015 ) was used to predict whether miR-506 targeted the 
Table 1 Association of miR-506 Expression with Clinicopathological Data from Gastric Cancer Patients

\begin{tabular}{|c|c|c|c|}
\hline Characteristics & $\begin{array}{l}\text { No. of } \\
\text { samples }\end{array}$ & $\begin{array}{l}\text { miR-506 high } \\
\text { expression } \\
\text { tumors, No. (\%) }\end{array}$ & $P$ value \\
\hline \multicolumn{4}{|l|}{ Sex } \\
\hline Male & 65 & $28(43.08)$ & \multirow[t]{2}{*}{0.236} \\
\hline Female & 44 & $14(31.82)$ & \\
\hline \multicolumn{4}{|l|}{ Age, years } \\
\hline$<50$ & 68 & $30(44.12)$ & \multirow[t]{2}{*}{0.821} \\
\hline$\geq 50$ & 41 & $19(46.34)$ & \\
\hline Primary tumors & 25 & $16(64.00)$ & \multirow[t]{2}{*}{$0.003^{*}$} \\
\hline $\begin{array}{l}\text { Metastasis (lymph node } \\
\text { and distant) }\end{array}$ & 84 & $26(30.95)$ & \\
\hline \multicolumn{4}{|l|}{ Tumor size, T stage } \\
\hline $\mathrm{T} 1$ & 19 & $11(57.89)$ & \multirow[t]{4}{*}{$0.041^{*}$} \\
\hline T2 & 28 & $12(42.86)$ & \\
\hline T3 & 42 & $13(30.95)$ & \\
\hline T4 & 20 & $6(30.00)$ & \\
\hline \multicolumn{4}{|c|}{ Lymph node metastasis, $\mathrm{N}$ stage } \\
\hline NO & 25 & $16(64.00)$ & \multirow[t]{4}{*}{$0.024^{*}$} \\
\hline N1 & 30 & $9(30.00)$ & \\
\hline N2 & 27 & $9(33.33)$ & \\
\hline N3 & 27 & $8(29.63)$ & \\
\hline \multicolumn{4}{|c|}{ Distant metastasis, M stage } \\
\hline MO & 101 & $40(39.60)$ & \multirow[t]{2}{*}{0.708} \\
\hline M1 & 8 & $2(25.00)$ & \\
\hline \multicolumn{4}{|l|}{ Clinical stage } \\
\hline I & 27 & $17(62.96)$ & \multirow[t]{4}{*}{$0.000^{\dagger}$} \\
\hline II & 27 & $12(44.44)$ & \\
\hline III & 47 & $11(23.40)$ & \\
\hline IV & 8 & $2(25.00)$ & \\
\hline
\end{tabular}

$* P<0.05$.

†Value obtained by SPSS analysis.

$3^{\prime}$-UTRs of ETS1. Mutations in the miR-506 target sites were generated. For the luciferase assay, cells $\left(2 \times 10^{5}\right)$ were seeded in triplicate in 6-well plates and allowed to settle for 24 hours. Different concentrations of miR-506 mutant, inhibitor, mimics, or the control-luciferase plasmid were synthesized at Kunming Medical University and RiboBio Co Ltd. Plasmids were transfected into gastric cancer cells using Lipofectamine 2000 reagent (Invitrogen), according to the manufacturer's recommendations. Luciferase signals were measured 48 hours after transfection using the Dual Luciferase Reporter Assay Kit (Promega, Madison, WI), according to the manufacturer's protocol. Three independent experiments were performed, and the data are presented as the means $\pm \mathrm{SD}$.

\section{Immunohistochemistry}

An EnVision detection kit (GK500705; Dako, Glostrup, Denmark) was used for immunohistochemical analysis of ETS1 and matrix metalloproteinase (MMP)-9 proteins in gastric cancer tissues, according to the manufacturer's protocol. Briefly, primary antibodies were diluted in phosphate-buffered saline, according to the manufacturers' recommendations: 1:50 for ETS1 (NCL-ETS-1; Novocastra,
Newcastle upon Tyne, UK) and 1:400 for MMP-9 (sc-21733; Santa Cruz Biotechnology Inc.). A total volume of $50 \mu \mathrm{L}$ diluted antibody solution was added to each section and incubated overnight at $4{ }^{\circ} \mathrm{C}$. The next day, after washing with phosphate-buffered saline, sections were incubated with Dako REAL EnVision/HRP (Dako Corp., Carpinteria, CA) for 30 minutes each. The color of the complexes was visualized by incubating tissue sections with a chromogenic substrate, 3,3'-diaminobenzidine (Dako Corp.) for 3 minutes, after which slides were rinsed in water, counterstained with hematoxylin, and mounted with Eukitt (O. Kindler GmbH \& Co, Freiburg, Germany). ETS1 and MMP-9 protein expression in gastric cancer tissue specimens, as detected by antibodies, was reviewed and scored under a light microscope by two independent pathologists
A
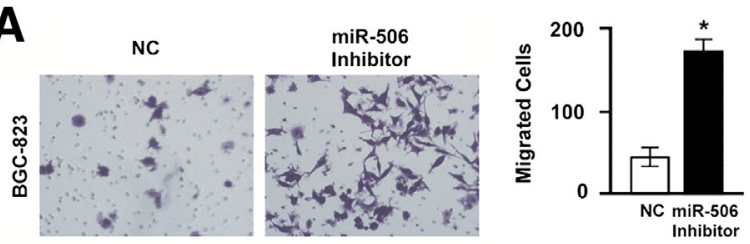

B
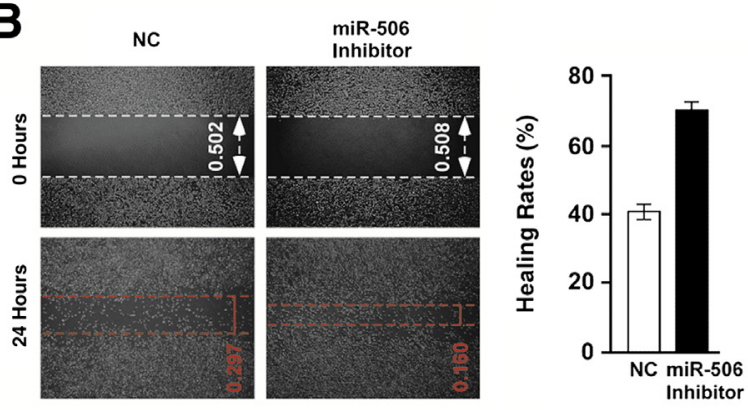

C

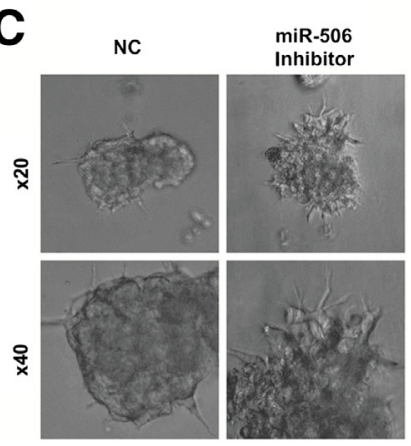

D

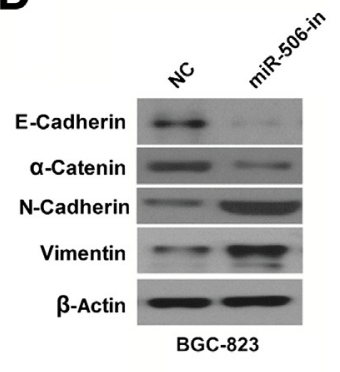

Figure 2 Regulation of epithelial-to-mesenchymal transition by miR506 overexpression. A: Matrigel invasion: SGC-7901 cells $\left(1 \times 10^{4}\right)$ were transfected with miR-506 mimics or negative control (NC), and the number of cells that invaded the basal side of the Matrigel-coated transwell inserts was counted after 24 hours. B: Wound-healing assay: cells from the same transfection as in A were seeded into 6-well plates, and a wound was generated at 24 hours after transfection. C: The pseudopodia growth of SGC-7901 cells from the same transfection as in $\mathbf{A}$ was assessed through three-dimensional cell culture. The cells were grown in a cell culture box for 10 to 14 days. D: Western blot analysis of the epithelial adherent markers $\mathrm{E}$-cadherin and $\alpha$-catenin, and mesenchymal markers $\mathrm{N}$-cadherin and vimentin, in the same SGC-7901-transfected cells as in A. Both blots were reprobed with $\beta$-actin to control for protein loading. Error bars represent means $\pm \mathrm{SD}(\mathbf{A}) . n=3$ to $7(\mathbf{A}) ; n=3(\mathbf{C}) .{ }^{*} P<0.05$. 

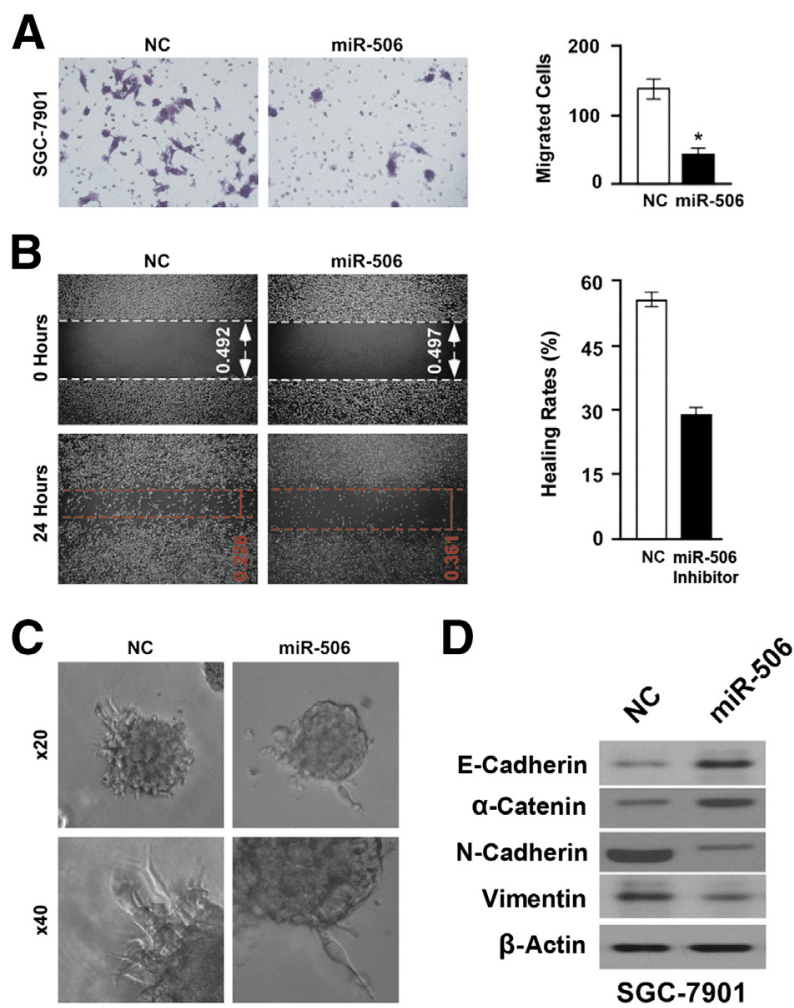

Figure 3 Regulation of epithelial-to-mesenchymal transition by miR506 inhibition. A: Matrigel invasion: BGC-823 cells $\left(1 \times 10^{4}\right)$ were transfected with miR-506 inhibitor or negative control (NC), and the number of cells that invaded the basal side of Matrigel-coated transwell inserts was counted after 24 hours. B: Wound healing assay: cells from the same transfection as in $\mathbf{A}$ were seeded into 6 -well plates, and a wound was applied at 24 hours after transfection. C: Pseudopodia growth of BGC-823 cells from the same transfection as in $\mathbf{A}$ was assessed through threedimensional cell culture. The cells were grown in a cell culture box for 10 to 14 days. D: Western blot analysis of the epithelial adherent markers $\mathrm{E}$-cadherin and $\alpha$-catenin, and mesenchymal markers $\mathrm{N}$-cadherin and vimentin, in the same BGC-823-transfected cells as in A. Both blots were reprobed with $\beta$-actin to control for protein loading. Error bars represent means $\pm \mathrm{SD}(\mathbf{A}) . n=3(\mathbf{A}$ and $\mathbf{C}) .{ }^{*} P<0.05$.

(Y.W. and S.D.) who were not aware of the clinicopathological data.

\section{Statistical Analysis}

All statistical analyses were performed using SPSS for Windows version 13.0 (SPSS Inc., Chicago, IL). The $t$-test was used to evaluate whether there was a significant difference between the two groups of data in all of the pertinent experiments. $P<0.05$ (using a two-tailed paired $t$-test) was considered statistically significant.

\section{Results}

High Expression of miR-506 Is Associated with Longer Survival Times of Gastric Cancer Patients

To observe whether miR-506 expression levels affected the clinical outcomes of gastric cancer patients, we used quantitative real-time PCR in a blinded manner to detect miR-506 expression levels in a cohort of human gastric cancer samples. Strikingly, miR-506 expression levels robustly stratified advanced clinical stage and early-stage patients (Figure 1A and Table 1). Patients whose primary gastric cancer lesions expressed high miR-506 levels (miR506 levels greater than the mean) exhibited significantly
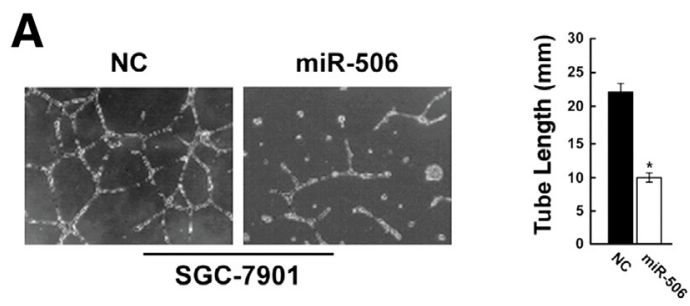

B
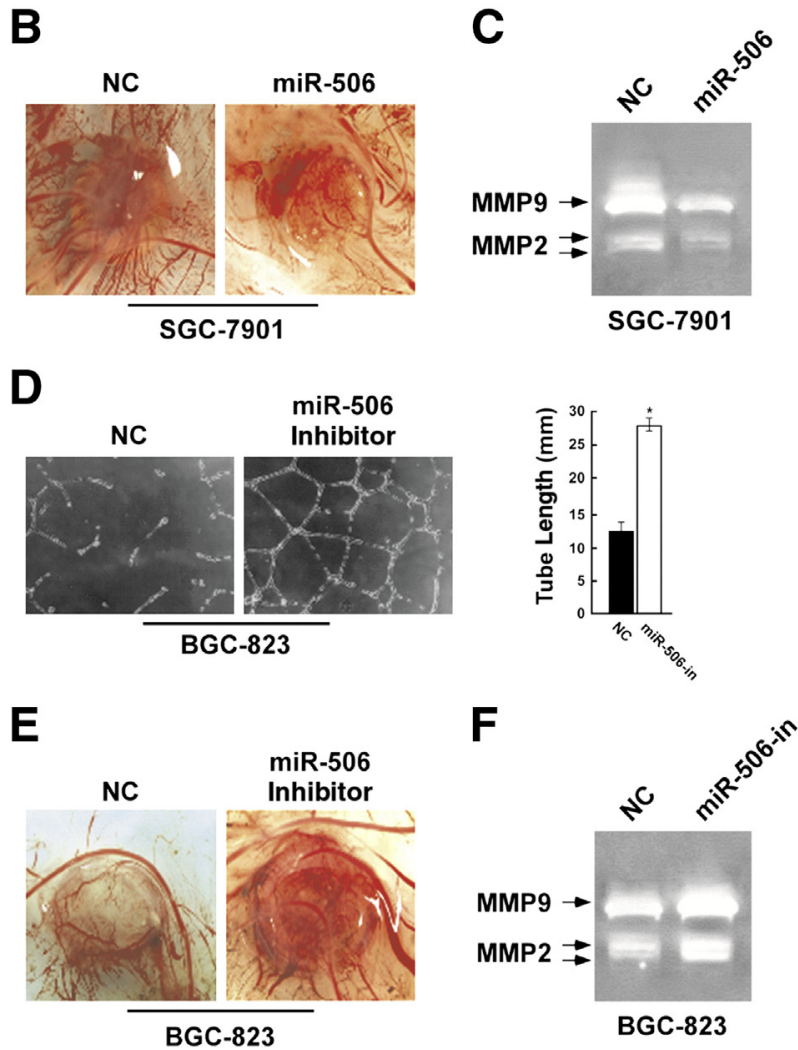

Figure 4 Angiogenesis regulation via miR-506. A: Matrigel-based tube formation assay assessed in SGC-7901 cells $\left(5 \times 10^{4}\right)$ transfected with miR-506 mimics or negative control (NC). The assay was quantified by the length of tubes after 6 hours. B: Vessel density was quantified by morphometric evaluation of chick chorioallantoic membrane (CAM) assay in SGC-7901 cells from the same transfection as in A. C: Gelatin zymography assay of matrix metalloproteinase (MMP)-9 and MMP-2 expression in SGC-7901 cells from the same transfection as in $\mathbf{A}$ shows that miR-506 expression is associated with decreased expression of MMP-9 and MMP-2. D: Matrigel-based tube formation assay assessed in BGC-823 cells $\left(5 \times 10^{4}\right)$ transfected with miR-506 inhibitor or control miRNA (NC). The assay was quantified by the length of tubes after 6 hours. E: Vessel density quantified by morphometric evaluation of CAM assay in BGC-823 cells from the same transfection as in D. F: Gelatin zymography assay of MMP-9 and MMP-2 expression in $\mathrm{BGC}-823$ cells from the same transfection as in $\mathbf{D}$ shows that miR-506 expression is associated with decreased expression of MMP-9 and MMP2. Error bars represent means $\pm \mathrm{SD}(\mathbf{A}$ and $\mathbf{D}) . n=3$ (A and $\mathbf{D}) ; n=5$ to 7 (F). ${ }^{*} P<0.05$. 
longer overall survival times than those whose primary lesions expressed low miR-506 levels (miR-506 levels less than the mean) (Figure 1B). We also examined the expression of miR-506 in seven gastric cancer cell lines and normal gastric tissues. Ubiquitous basal miR-506 expression was lower in gastric cancer cells than in normal gastric tissues. In addition, miR-506 expression was closely associated with the invasion properties of gastric cancer cells (Figure 1, C and D). The SGC-7901 cell line showed the lowest miR-506 expression and the highest invasive ability, whereas the BGC-823 cell line showed the highest miR-506 expression and the lowest invasive ability of all of the gastric cancer cell lines examined. Because of these divergent results, the SGC-7901 and BGC-823 cell lines were chosen for further miR-506 knock-in and knockdown experiments.

\section{miR-506 Inhibits the EMT of Gastric Cancer Cells}

Because epithelial-to-mesenchymal transition (EMT) is an important process that enables cancer cells to invade their surroundings and metastasize, we examined whether miR-506 overexpression correlated with EMT in human gastric cancer cell lines. We observed, as expected, that miR-506 overexpression reduced the ability of SGC-7901 cells to invade through Matrigel (Figure 2A). In addition, wound-healing assays revealed that the migration ability of SGC-7091 cells was diminished (Figure 2B). These results indicated that miR-506 could be a potential suppressor of gastric cancer metastasis. Furthermore, the growth of gastric cancer cell pseudopodia is considered a prometastatic phenotype, and this was robustly decreased by miR-506 overexpression (Figure 2C). Consistent with this, the expression of E-cadherin and $\alpha$-catenin was elevated, and the expression of $\mathrm{N}$-cadherin and vimentin was decreased by miR-506 overexpression (Figure 2D), suggesting that miR-506 acts as an epithelial transition promoter in gastric cancer cells.

Consistent with the miR-506 overexpression results, miR506 inhibitors significantly increased the invasive (Figure 3A) and motive (Figure 3B) ability of BGC-823 cells. Furthermore, miR-506 suppression increased the growth of gastric cancer cell pseudopodia (Figure 3C).
Similarly, Western blot analysis showed that miR-506 inhibition caused decreased expression of E-cadherin and $\alpha$-catenin, as well as increased expression of $\mathrm{N}$-cadherin and vimentin (Figure 3D). Collectively, these findings indicate that miR-506 plays a role in EMT suppression in gastric cancer cells.

\section{miR-506 Suppresses Angiogenesis in Gastric Cancer}

Because angiogenesis is an important factor in cancer metastasis, we examined the angiogenic effects of miR-506 expression in gastric cancer cells. A Matrigel-based tube formation assay using SGC-7901 cells showed that miR-506 overexpression suppressed vascular tubule development (Figure 4A). Accordingly, as quantified by histological morphometric evaluation of CAM sections, vessel density was significantly reduced in SGC-7901 cells with miR-506 overexpression (Figure 4B). Consistent with this, gelatin zymography assays showed that the expression levels of MMP-9 and MMP-2 were decreased by miR-506 overexpression (Figure 4C). This suggests that miR-506 contributes to extracellular matrix maintenance in the gastric cancer microenvironment. Conversely, miR-506 inhibition in BGC-823 cells promoted the formation of a tubular polygonal network (Figure 4D) and induced vessel growth on the CAM (Figure 4E). Furthermore, gelatin zymography assays showed that miR-506 expression inhibition was associated with the expression of MMP-9 and MMP-2 (Figure 4F). These findings indicate that miR-506 is necessary and sufficient for angiogenesis suppression during gastric cancer progression.

\section{ETS1 Is a Direct Target of miR-506 Regulation}

We next investigated the direct molecular targets of miR506. The miR-506 target genes were predicted and selected using TargetScan software. On the basis of our analysis, we found that miR-506 targets the $3^{\prime}$-UTRs of ETS1 (Figure 5A). The expression levels of ETS1 and MMP-9 were lower in miR-506-transfected SGC-7901 cells; however, their expression was augmented after miR-506 inhibition in BGC-823 cells (Figure 5B). To confirm miR-506
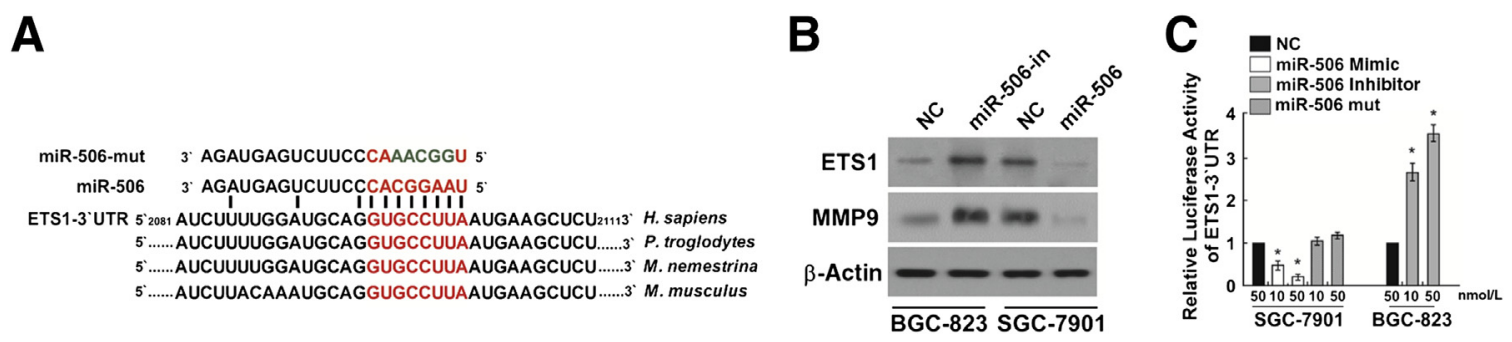

Figure 5 Identification of ETS1 as a direct target gene of miR-506. A: miR-506 targets the $3^{\prime}$-untranslated region (UTR) of ETS1 of multiple species, such as Homo sapiens, Pan troglodytes, Macaca nemestrina, and Mus musculus. B: miR-506 was overexpressed or inhibited in BGC-823 and SGC-7901 gastric cancer cell lines, respectively, to monitor the expression of ETS1 and matrix metalloproteinase (MMP)-9 by Western blot analysis. C: Luciferase activity of ETS1 3'-UTR in SGC-7901 and BGC- 823 cells transfected with control miRNA [negative control (NC)], miR-506 mimics, or miR-506 inhibitors. Error bars represent means \pm SD (C). $n=3(\mathbf{C}) .{ }^{*} P<0.05$. 

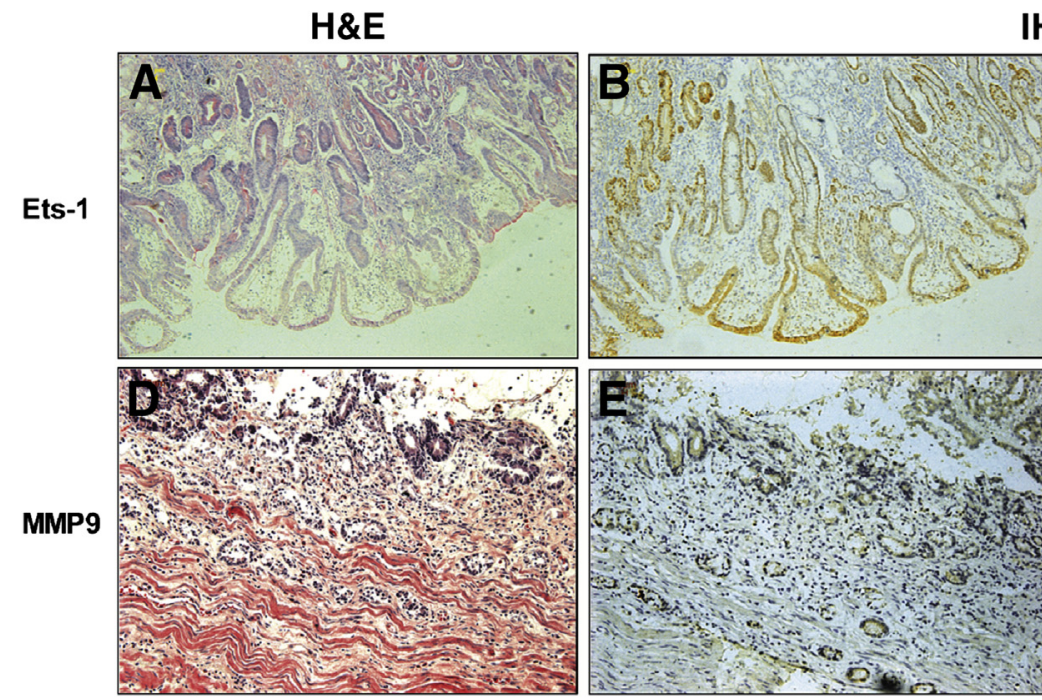

IHC

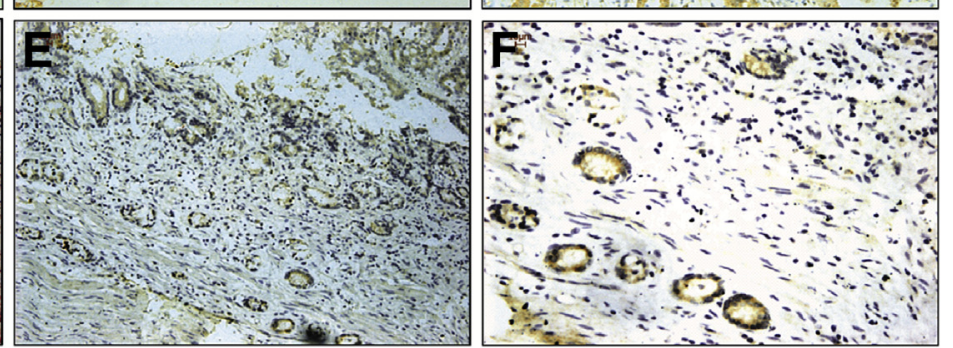

Figure 6 Expression of ETS1 and matrix metalloproteinase (MMP)-9 proteins in gastric cancer tissues. Gastric cancer tissue specimen for primary antibodies to ETS1 stained with hematoxylin and eosin (H\&E; objective magnification of $\times 5 ; \mathbf{A})$, primary antibodies to ETS1 (objective magnification of $\times 5$; B), primary antibodies to ETS1 (objective magnification of $\times 10 ; \mathbf{C}$ ), primary antibodies to MMP-9 stained with H\&E (objective magnification of $\times 5$; D), primary antibodies for MMP-9 (objective magnification of $\times 5 ; \mathbf{E}$ ), and primary antibodies for MMP-9 (objective magnification of $\times 10 ; \mathbf{F}$ ). The data in $\mathbf{C}$ show positive nuclear and in $\mathbf{F}$ show positive cytoplasmic staining. IHC, immunohistochemistry.

interaction with the $3^{\prime}$-UTR of ETS1, wild-type or mutant miR-506-binding sequences from the $3^{\prime}$-UTR of the ETS1 gene were cloned into the $3^{\prime}$-UTR of the luciferase reporter gene for luciferase assays. Overexpression of miR-506 with the wild-type ETS1 3'-UTR in SGC-7901 and BGC-823 cells resulted in a significant decrease in luciferase activity; however, luciferase activity was not decreased by overexpression of miR-506 with mutant 3'-UTR miR506-binding sequences (Figure 5C). Collectively, these results indicate that ETS1 may be a potential target mediating miR-506-dependent regulation in gastric cancer cells.

\section{The Expression of ETS1 Is Associated with MMP-9 in Gastric Cancer Tissues}

Because MMP-9 is known to participate in gastric cancer metastasis and ETS1 was reported to be an MMP9 transcription factor, we examined the expression of both proteins in gastric cancer tissues. Protein expression levels were examined in a cohort of human gastric cancer samples in a blinded manner. Nuclear accumulation of ETS1 was observed in $71.10 \%(123 / 173)$ of the gastric cancer tissues examined (Figure 6, A-C), whereas $75.14 \%$ (130/173) of the tissue samples examined stained positively for MMP-9 (Figure 6, D-F). ETS1 expression was associated with MMP-9 expression $(r=0.459, P \leq 0.0001)$ (Table 2), indicating that ETS1 and MMP-9 synergistically regulate gastric cancer metastasis.

\section{Discussion}

EMT and angiogenesis are two essential processes in cancer progression. ${ }^{16,17}$ In this study, we report a significant role for miR-506 in gastric cancer EMT and angiogenesis suppression. In addition, we demonstrated that higher endogenous miR-506 expression was associated with longer survival times in gastric cancer patients. miR-506 is located at $\mathrm{Xq} 27.3$, a chromosomal region closely associated with fragile $\mathrm{X}$ syndrome. ${ }^{18}$ Down-regulation of miR-506 has been detected in several solid tumors, including serous ovarian cancer and breast cancer, ${ }^{19,20}$ suggesting that miR506 plays an important role in tumor suppression. Our study presents evidence that miR-506 is a potent inhibitor of EMT and that miR-506 overexpression is associated with decreased vimentin and increased E-cadherin expression in gastric cancer. We also explored the potential role of miR506 in angiogenesis and found that miR-506 both suppresses angiogenesis in gastric cancer and is associated with decreased MMP-9 expression. These findings suggest that miR-506 acts as a suppressor of angiogenesis and metastasis in gastric cancer.

We also demonstrated that ETS1 is a direct target of miR506. The necessity of ETS1 for endothelial cells to adopt an angiogenic, blood vessel-forming phenotype has been well documented. $^{21-23}$ Accordingly, ETS1 is abundant in regenerating adult tissues and in areas of the developing embryo that require the formation of new blood vessels. ${ }^{24,25}$

Table 2 The Relationship between ETS1 and MMP-9 in Gastric Cancer Tissues

\begin{tabular}{llrll}
\hline & \multicolumn{4}{l}{ ETS1, No. $(\%)$} \\
\cline { 2 - 5 } MMP-9 & Negative & Positive & $P$ value & R \\
\hline Negative $(n=43)$ & $28(65.12)$ & $15(34.88)$ & 0.000 & 0.459 \\
Positive $(n=130)$ & $22(16.92)$ & $108(83.08)$ & & \\
\hline
\end{tabular}

MMP, matrix metalloproteinase. 
Because the acquisition of invasive behavior is a part of the endothelial activation program, ETS1 may be responsible for stimulating proteases necessary for these processes. ${ }^{26,27}$ Likewise, ETS1 may help cancer cells get nutrients and oxygen by inducing tumor vascularization, and may promote tumor metastasis and invasion by activating ECMdegrading proteases in tumor or mesenchymal cells. ${ }^{28}$ Thus, high ETS1 levels in tumors often correlate with a poorer prognosis. ${ }^{22}$

ETS1 was reported to be an MMP-9 transcription factor. ${ }^{29}$ MMP-9 plays a critical role in promoting tumor progression by degrading the extracellular matrix and altering cell adhesion. ${ }^{30}$ In this study, immunohistochemistry of clinical gastric cancer samples showed that ETS1 expression was associated with MMP-9 expression, indicating that ETS1 and MMP-9 synergistically regulate gastric cancer metastasis. Thus, it is possible that a miR-506-ETS1 axis plays an important role in the regulation of gastric cancer angiogenesis.

In summary, cancer is a complex disease and controlling cancer development and progression requires system-level and integrative approaches. Our study revealed the functional relevance of miR-506 with respect to angiogenesis and metastasis, suggesting that miR-506 acts as a tumor suppressor in gastric cancer. Additional studies will be needed to explore the potential clinical utility of miR-506 as a potential biomarker for gastric cancer prognosis and as a new potential therapeutic target.

\section{Acknowledgments}

Z.L. performed cellular function studies and drafted the manuscript. Z.L. and S.D. performed cellular studies and statistical analysis and wrote the manuscript. J.Z. and J.T. collected the clinical tissue samples. J.Y. communicated and followed up with patients. Y.W. and M.L. performed immunohistochemistry. C.G., R.L., and Y.X. performed cell culture and protein extraction. W.W., X.X., and T.G. analyzed clinical samples. H.D. performed real-time PCR. K.Y. designed the study. X.S. conceived, designed, and coordinated the study. All authors read and approved the final manuscript.

\section{References}

1. Siegel R, Naishadham D, Jemal A: Cancer statistics, 2013. CA Cancer J Clin 2013, 63:11-30

2. Herszenyi L, Tulassay Z: Epidemiology of gastrointestinal and liver tumors. Eur Rev Med Pharmacol Sci 2010, 14:249-258

3. Brenner H, Rothenbacher D, Arndt V: Epidemiology of stomach cancer. Methods Mol Biol 2009, 472:467-477

4. Chen WQ, Zheng RS, Zhang SW, Zeng HM, Zou XN: The incidences and mortalities of major cancers in China, 2010. Chin J Cancer 2014, 33:402-405

5. Kusano T, Shiraishi N, Shiroshita H, Etoh T, Inomata M, Kitano S: Poor prognosis of advanced gastric cancer with metastatic suprapancreatic lymph nodes. Ann Surg Oncol 2013, 20:2290-2295
6. Berindan-Neagoe I, Monroig Pdel C, Pasculli B, Calin GA: MicroRNAome genome: a treasure for cancer diagnosis and therapy. CA Cancer J Clin 2014, 64:311-336

7. He L, Hannon GJ: MicroRNAs: small RNAs with a big role in gene regulation. Nat Rev Genet 2004, 5:522-531

8. Bartel DP: MicroRNAs: target recognition and regulatory functions. Cell 2009, 136:215-233

9. Filipowicz W, Bhattacharyya SN, Sonenberg N: Mechanisms of posttranscriptional regulation by microRNAs: are the answers in sight? Nat Rev Genet 2008, 9:102-114

10. Bagga S, Bracht J, Hunter S, Massirer K, Holtz J, Eachus R, Pasquinelli AE: Regulation by let-7 and lin-4 miRNAs results in target mRNA degradation. Cell 2005, 122:553-563

11. Huang Q, Gumireddy K, Schrier M, le Sage C, Nagel R, Nair S, Egan DA, Li A, Huang G, Klein-Szanto AJ, Gimotty PA, Katsaros D, Coukos G, Zhang L, Pure E, Agami R: The microRNAs miR-373 and miR-520c promote tumour invasion and metastasis. Nat Cell Biol 2008, 10:202-210

12. Helwak A, Kudla G, Dudnakova T, Tollervey D: Mapping the human miRNA interactome by CLASH reveals frequent noncanonical binding. Cell 2013, 153:654-665

13. Lin Z, Liu Y, Sun Y, He X: Expression of Ets-1, Ang-2 and maspin in ovarian cancer and their role in tumor angiogenesis. J Exp Clin Cancer Res 2011, 30:31

14. von Thun A, Birtwistle M, Kalna G, Grindlay J, Strachan D, Kolch W, von Kriegsheim A, Norman JC: ERK2 drives tumour cell migration in three-dimensional microenvironments by suppressing expression of Rab17 and liprin-beta2. J Cell Sci 2012, 125: $1465-1477$

15. Washington K: 7th Edition of the AJCC cancer staging manual: stomach. Ann Surg Oncol 2010, 17:3077-3079

16. Noncanonical FZD2 signaling induces EMT and tumor progression. Cancer Discov 2015, 5:Of17

17. Bonapace L, Coissieux MM, Wyckoff J, Mertz KD, Varga Z, Junt T, Bentires-Alj M: Cessation of CCL2 inhibition accelerates breast cancer metastasis by promoting angiogenesis. Nature 2014, 515:130-133

18. Grabczyk E, Kumari D, Usdin K: Fragile X syndrome and Friedreich's ataxia: two different paradigms for repeat induced transcript insufficiency. Brain Res Bull 2001, 56:367-373

19. Yang D, Sun Y, Hu L, Zheng H, Ji P, Pecot CV, Zhao Y, Reynolds S, Cheng H, Rupaimoole R, Cogdell D, Nykter M, Broaddus R, Rodriguez-Aguayo C, Lopez-Berestein G, Liu J, Shmulevich I, Sood AK, Chen K, Zhang W: Integrated analyses identify a master microRNA regulatory network for the mesenchymal subtype in serous ovarian cancer. Cancer Cell 2013, 23: 186-199

20. Arora H, Qureshi R, Park WY: miR-506 regulates epithelial mesenchymal transition in breast cancer cell lines. PLoS One 2013, 8:e64273

21. Naito S, Shimizu S, Matsuu M, Nakashima M, Nakayama T, Yamashita S, Sekine I: Ets-1 upregulates matrix metalloproteinase1 expression through extracellular matrix adhesion in vascular endothelial cells. Biochem Biophys Res Commun 2002, 291: $130-138$

22. Dittmer J: The biology of the Ets1 proto-oncogene. Mol Cancer 2003, 2:29

23. Arderiu G, Pena E, Badimon L: Angiogenic microvascular endothelial cells release microparticles rich in tissue factor that promotes postischemic collateral vessel formation. Arterioscler Thromb Vasc Biol 2015, 35:348-357

24. Zhu W, Yao X, Liang Y, Liang D, Song L, Jing N, Li J, Wang G: Mediator Med23 deficiency enhances neural differentiation of murine embryonic stem cells through modulating BMP signaling. Development 2015, 142:465-476

25. Simoes-Costa M, Tan-Cabugao J, Antoshechkin I, Sauka-Spengler T, Bronner ME: Transcriptome analysis reveals novel players in the 
cranial neural crest gene regulatory network. Genome Res 2014, 24 281-290

26. Sato Y, Teruyama K, Nakano T, Oda N, Abe M, Tanaka K, IwasakaYagi C: Role of transcription factors in angiogenesis: Ets-1 promotes angiogenesis as well as endothelial apoptosis. Ann N Y Acad Sci 2001, 947:117-123

27. Zhang Y, Yan LX, Wu QN, Du ZM, Chen J, Liao DZ, Huang MY, Hou JH, Wu QL, Zeng MS, Huang WL, Zeng YX, Shao JY: miR-125b is methylated and functions as a tumor suppressor by regulating the ETS1 proto-oncogene in human invasive breast cancer. Cancer Res 2011, 71:3552-3562
28. Okano K, Hibi A, Miyaoka T, Inoue T, Sugimoto H, Tsuchiya K, Akiba T, Nitta K: Inhibitory effects of the transcription factor Ets-1 on the expression of type I collagen in TGF-beta1-stimulated renal epithelial cells. Mol Cell Biochem 2012, 369:247-254

29. Ghosh S, Basu M, Roy SS: ETS-1 protein regulates vascular endothelial growth factor-induced matrix metalloproteinase- 9 and matrix metalloproteinase-13 expression in human ovarian carcinoma cell line SKOV-3. J Biol Chem 2012, 287:15001-15015

30. Gialeli C, Theocharis AD, Karamanos NK: Roles of matrix metalloproteinases in cancer progression and their pharmacological targeting. FEBS J 2011, 278:16-27 